\title{
Dual enantioselective control by heterocycles of (S)-indoline derivatives*
}

\author{
Yong Hae Kim${ }^{\ddagger}$, Doo Young Jung, So Won Youn, Sam Min Kim, and \\ Doo Han Park
}

\begin{abstract}
Center for Molecular Design and Synthesis, Department of Chemistry, Korea Advanced Institute of Science and Technology, Taejon 305-701, Korea
\end{abstract}

\begin{abstract}
Diastereo-and enantioselective pinacol coupling reactions of chiral $\alpha$-ketoamides mediated by samarium diiodide afforded extremely high diastereoselectivities. Enantiopure $(S, S)$ - or $(R, R)$-2,3-dialkyltartaric acid and derivatives can be synthesized. Diels-Alder cycloadditions of $S$-indoline chiral acrylamides with cyclopentadiene in the presence of Lewis acids proceed with high diastereofacial selectivity, giving either endo- $R$ or endo-S products depending on Lewis acid and the structures of chiral dienophiles.
\end{abstract}

Keywords: Enantioselective; pinacol coupling; $\alpha$-ketoamides; samarium diiodide; $S$-indoline.

In chemical transformations, the synthesis of individual enantiomers is generally achieved by using natural chiral sources. Because sometimes natural sources of one of the enantiomers may be limited, it is desirable and important to obtain both enantiomers by stereocontrolled reactions of prochiral compounds that utilize the same chiral source. Only a few examples have been reported where the same reaction system gives both enantiomers depending on reaction conditions. ( $S$ )-Indoline-2-carboxylic acid has been found to be one of the best starting materials for the synthesis of the new chiral materials, especially for obtaining both $R$ - and $S$-enantiomers from asymmetric reactions starting from the same starting material [1]. There are several examples of production of both diastereomers or enantiomers with high stereoselectivity using chiral catalysts or ligands derived from the same starting material of $(S)$-indoline-2-carboxylic acid [2,3].

The intermolecular coupling of various aldehydes or ketones to the corresponding pinacols has been extensively studied. However, enantiopure pinacols have not really been obtained using this type of coupling [4]. To obtain chiral 2,3-dialkyltartaric acids, which can be utilized in design of chiral ligands or chiral auxiliaries, we studied the pinacol coupling reaction of chiral $\alpha$-ketoamides 1 with $\mathrm{SmI}_{2}$ to obtain pinacol product $\mathbf{2}$ in extremely high diastereoselectivities.

$\alpha$-Ketoamides 1 in the presence of $\mathrm{SmI}_{2}, \mathrm{HMPA}$, and $t \mathrm{BuOH}$ in tetrahydrofuran (THF) dimerized to give pinacol 2 with extremely high diastereoselectivity ( $>98 \%$ de in some cases; Scheme 1). Also, we have found that $(2 S, 3 \mathrm{a} S, 7 \mathrm{a} S)-N$-pyruvoyl-2-(tert-butyldiphenylsilyloxy)octahydroindoline $\mathbf{1 a}$ ' (99.8\% ee), affords the opposite configuration of 3a' ( $R, R$ diol: $97 \%$ de). The ratio of $R, R: S, S: m e s o$ is 98.5: 0.5:1.5 [2].

\footnotetext{
*Paper based on a presentation at the $7^{\text {th }}$ IUPAC International Conference on Heteroatom Chemistry (ICHAC-7), Shanghai, China, 21-25 August 2004. Other presentations are published in this issue, pp. 1985-2132.

¥Corresponding author
} 


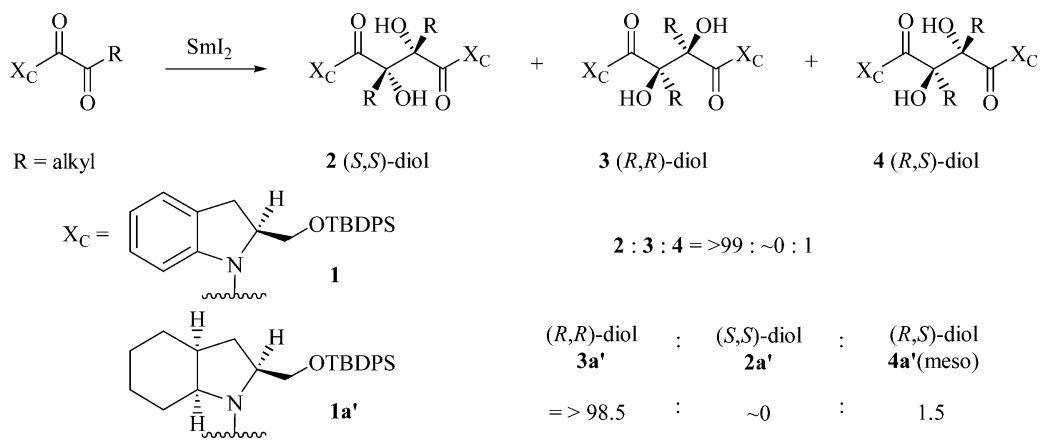

Scheme 1

The reaction appears to be initiated by formation of a ketyl radical $\mathbf{A}$ with a one-electron transfer to $\mathrm{SmI}_{2}$ followed by coupling of the two ketyl radicals (Fig. 1). The coupling takes place at the less hindered si face and gives high diastereoselectivities. The different configurations of the adducts produced can be rationalized by the different intermediates formed from $\mathbf{1}$ and $\mathbf{1 a}$ ' with $\mathrm{SmI}_{2}$. Instead of $\mathbf{A}$, the intermediate $\mathbf{A}^{\prime}$ is favorable in the case of $\mathbf{1} \mathbf{a}^{\prime}$ more so than $\mathbf{A}^{\prime \prime}$ due to the steric repulsion between the chair conformation of the cyclohexane ring and the amide carbonyl moiety (Fig. 1). When the reaction was performed with $l$-proline-derived chiral $\alpha$-ketoamides, only poor selectivity was observed, indicating that the phenyl ring of indoline-2-carboxylic acid helped $\mathbf{A}$ to adopt the depicted conformation.

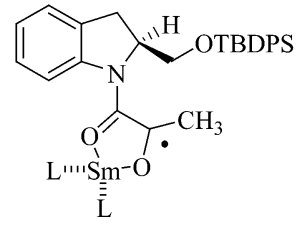

A

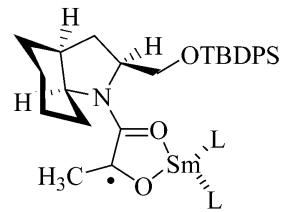

$\mathbf{A}^{\prime}$

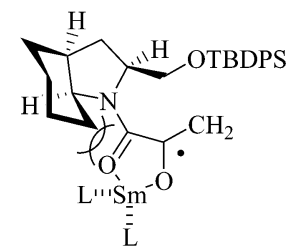

$\mathbf{A}^{\prime \prime}$

Fig. 1

In connection with the pinacol coupling reaction, $\alpha, \beta$-unsaturated amides have been found to react with $\mathrm{SmI}_{2}$ to form dimerized products containing two chiral carbons which are first obtained as the adjacent chiral hydrocarbons (Scheme 2).

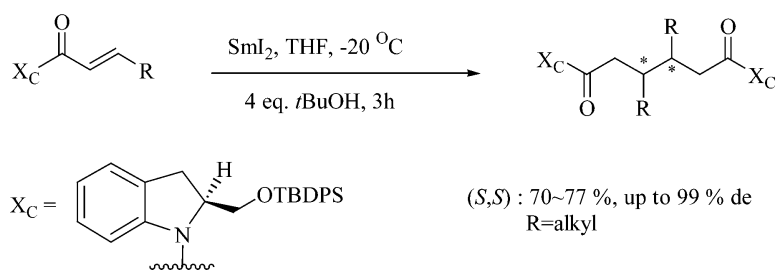

Scheme 2

In the Lewis acid-catalyzed Diels-Alder reactions of chiral dienophiles, generally the $S$-form of the chiral dienophile (auxiliary) exclusively affords the endo- $R$ adduct over the endo-S one, and the $R$-form exclusively gives the $S$-adduct over the endo-R one [5]. In the hope of obtaining the opposite configuration of the endo adduct and understanding the mechanism, three different dienophiles 6, 7, and 


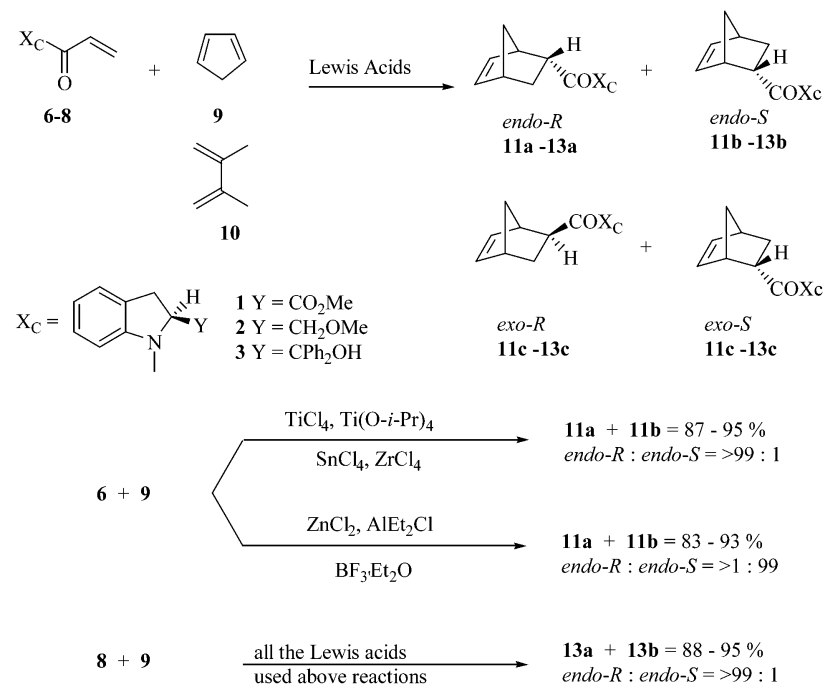

Scheme 3

$\mathbf{8}$ were prepared and reacted with dienes $\mathbf{9}$ and $\mathbf{1 0}$ in the presence of various Lewis acids, as shown in Scheme 3 [3].

Extremely high levels of asymmetric induction can be achieved in Diels-Alder cycloadditions of $\mathbf{6}$ or $\mathbf{8}$ with $\mathbf{9}$; in contrast to other general dienophiles, $\mathbf{6}$ containing a carboxylate moiety reacts with 9 to give differently configured adducts depending on the Lewis acids employed; in the presence of $\mathrm{TiCl}_{4}$, $\mathrm{Ti}\left(\mathrm{OPr}^{i}\right)_{4}$, or $\mathrm{SnCl}_{4}, \mathbf{1 1 a}$ was obtained as the major diastereomer $(1 \mathbf{1 a}: 11 \mathbf{b}=$ endo- $R$ :endo- $S=>99: 1)$, but with $\mathrm{AlEt}_{2} \mathrm{Cl}, \mathrm{ZnCl}_{2}$, or $\mathrm{BF}_{3} \cdot \mathrm{Et}_{2} \mathrm{O}$, the opposite configuration of $10 \mathbf{b}$ was obtained $(\mathbf{1 1 a}: 11 \mathbf{b}=$ 1:>99). In particular, 8 containing a diphenyl-substituted tertiary alcohol moiety affords exceptionally high diastereofacial selectivities $(\mathbf{1 3 a}: \mathbf{1 3 b}=>99: 1$, yield $=>90 \%)$ regardless of the nature of the Lewis acid.

The differently configured adducts produced can be rationalized by different intermediates formed between 6-8 and the metals of the Lewis acids. Compounds $6-8$ react with 9 to favor formation of endo-R species 11a or 13a with $\mathrm{TiCl}_{4}, \mathrm{Ti}\left(\mathrm{OPr}^{i}\right)_{4}, \mathrm{SnCl}_{4}$, or $\mathrm{ZrCl}_{4}$ probably via formation of sevenmembered ring chelates with the acryloyl moiety of $\mathbf{1 5}$ or $\mathbf{1 6}$ having a cisoid conformation (Fig 2). On the other hand, $\mathbf{6}$ or $\mathbf{7}$ prefer endo-S formation $\mathbf{1 1 b}$ or $\mathbf{1 2 b}$ with $\mathrm{ZnCl}_{2}, \mathrm{AlEtCl}_{2}$, or $\mathrm{BF}_{3} \cdot \mathrm{Et}_{2} \mathrm{O}$, with high diastereofacial selectivity probably resulting from intermediate 14, as shown in Fig. 2. Species 6 and 8 also reacted with less reactive acyclic diene 10 at $25{ }^{\circ} \mathrm{C}$ to result in the same trend: for $\mathbf{6}$ with $\mathrm{TiCl}_{4}$ the ratio of endo-R:endo-S was 97:3, while with $\mathrm{EtAlCl}_{2}$ the ratio was reversed to 3:97, for 8 with both $\mathrm{TiCl}_{4}$ and $\mathrm{Et}_{2} \mathrm{AlCl}$, endo-R:endo- $S=97: 3$ and 94:6, respectively.

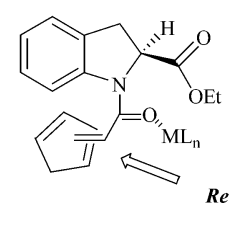

14 $\mathrm{M}=\mathrm{Zn}, \mathrm{Al}, \mathrm{B}$

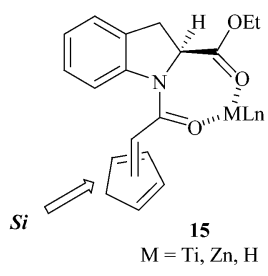

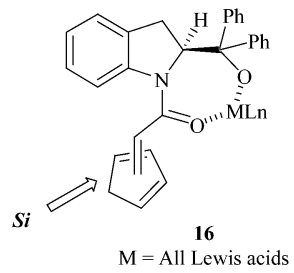

Fig. 2 
There are also examples of employing $(S)$-indoline-2-carboxylic acid-derived amino alcohols as chiral catalysts in asymmetric reactions [6,7]. Asymmetric reduction of ketones by amino alcohol-borane systems and asymmetric alkylation of aldehydes using dialkyl zinc reagents and amino alcohol catalysts are those examples. In both of the reactions, by just slight modification of indoline moiety, dual enantioselective control was achieved.

Numerous $\beta$-amino alcohols were tested as chiral ligands for the enantioselective reduction of ketones. Although several efficient catalysts have been developed, most of the oxazaborolidine catalysts derived from $l$-amino acids give access to only one enantiomer of secondary alcohols; namely, $(S)$-amino alcohol-borane systems have been reported to give arylalkyl alcohols with the $(R)$-configuration and $(R)$-amino alcohol-borane systems give $(S)$-arylalkyl alcohol, respectively [8]. However, in contrast to $l$-amino acids, which are readily available from natural sources, enantiopure $d$-amino acids are often difficult to obtain.

When the new $\beta$-amino alcohols 17, 18 are employed as catalysts in the asymmetric reduction of ketones, a remarkable reversal of enantiofacial selectivity is demonstrated [6] (Scheme 4). When acetophenone was reduced in the presence of catalytic amount $(10 \mathrm{~mol} \%)$ of $\mathbf{1 7 b},(R)$-phenylethanol of high optical purity was obtained. On the other hand, when the catalyst was $\mathbf{1 8 a}$, phenylethanol of opposite configuration $[(S)$-phenylethanol] was obtained in comparable optical purity. The chiral catalysts could be recycled without any significant loss of activity or optical purity. The same trend remained through other substrates tested, including even aliphatic ketone.

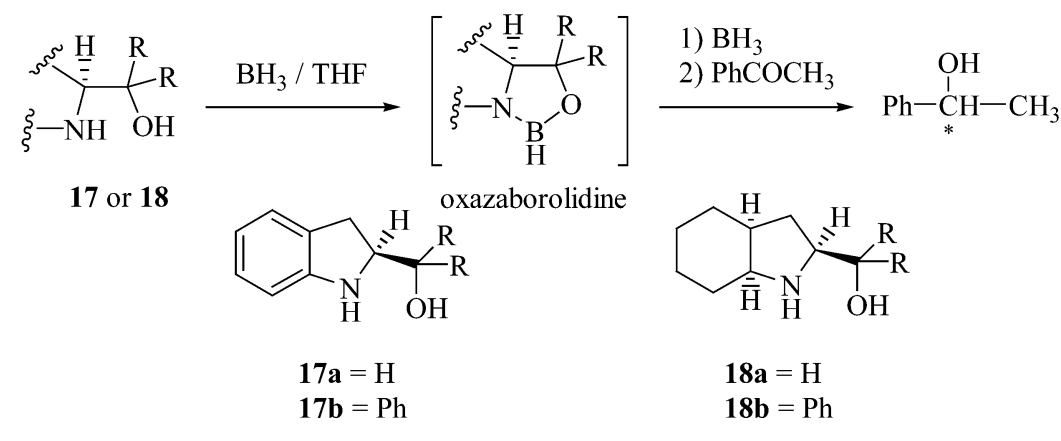

\begin{tabular}{ccccc}
\hline amino alcohol & $\begin{array}{l}\text { equiv. } \\
\text { (oxazaborolidine) }\end{array}$ & yield (\%) & e.e. (\%) & config. \\
\hline $\mathbf{1 7 a}$ & 0.1 & 96 & 82 & $R$ \\
$\mathbf{1 7 b}$ & 0.1 & 94 & 96 & $R$ \\
$\mathbf{1 8 a}$ & 0.1 & 95 & 90 & $S$ \\
$\mathbf{1 8 b}$ & 0.1 & 96 & 49 & $S$ \\
\hline
\end{tabular}

\section{Scheme 4}

The following model can rationalize the obtained results. In the presence of catalyst $\mathbf{1 7} \mathbf{b}$, the transition state $\mathbf{B}$ is favored the competing transition state $\mathbf{B}$ ' due to the steric effect of diphenyl group (Fig. 3). In this case, the phenyl ring of indoline moiety does not exert significant steric interactions in the oxazaborolidine-ketone-borane complex. On the contrary, in the case of 18a, the steric effect of the cyclohexyl group appears to enforce the approach of chiral oxazaborolidine complex to the opposite face of carbonyl compounds as in the transition state $\mathbf{C}$. The transition state $\mathbf{C}$ is favored over $\mathbf{C}^{\prime}$, because the cyclohexyl group from the oxazaborolidine repels the methyl or other $\mathrm{R}_{\mathrm{S}}$ of substrates. These 


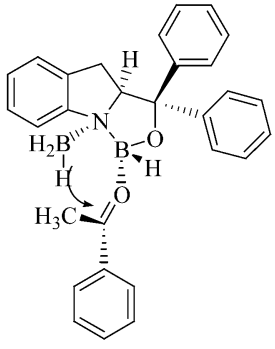

B

favorable

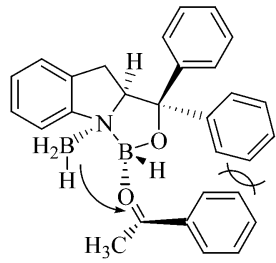

unfavorable
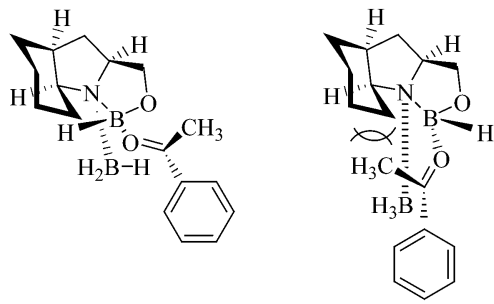

C
favorable
$C^{\prime}$

unfavorable

Fig. 3

transition states are well supported by the well-established fact that Lewis acidic boron atom favors the coordination of ketone with the large substituent $\mathrm{R}_{\mathrm{L}}$ anti to itself [9].

Despite numerous extensive studies regarding the asymmetric addition of organometallic reagents to aldehydes using chiral ligands [10], most of the chiral catalysts derived from one enantiomer of the natural or synthetic chiral sources, only allow the preparation of only one enantiomer of products. For example, $\beta$-amino alcohol catalysts of $(S)$-configuration produce only $(S)$-configured secondary alcohols in dialkyl zinc addition reactions. Because it is very important in good asymmetric catalysis that preparation of both of the enantiomers is efficiently achieved from the chiral catalysts derived from the same natural sources, the new chiral amino alcohols 17, 18 were again employed in the asymmetric alkylation of various aldehydes.

Again in this case, the remarkable reversal of enantiofacial selectivity was observed [7]. In the amino alcohol-catalyzed diethyl zinc addition to benzaldehyde, $\mathbf{1 7 b}$ catalyzed the addition to give $(S)$-phenylethanol in $96 \%$ e.e., whereas 18a catalyzed to give $(R)$-phenylethanol in $90 \%$ e.e. Also, they showed excellent ability to work as chiral catalysts against other aldehydes, including cinnamaldehyde and aliphatic aldehydes.
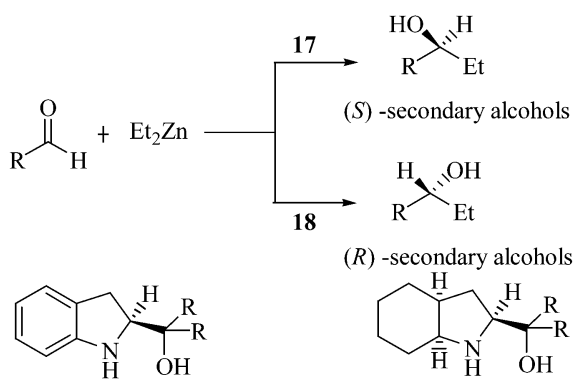

R) -secondary alcohols

\begin{tabular}{|c|c|c|c|}
\hline \multicolumn{2}{|c|}{$\begin{array}{l}17 \mathbf{a}=\mathrm{H} \\
17 \mathbf{b}=\mathrm{Ph}\end{array}$} & \multicolumn{2}{|c|}{$\begin{array}{l}18 \mathbf{a}=H \\
18 b=P h\end{array}$} \\
\hline amino alcohol & yield $(\%)$ & e.e. $(\%)$ & config. \\
\hline $17 \mathbf{a}$ & 96 & 72 & $S$ \\
\hline $17 b$ & 94 & 96 & $S$ \\
\hline $18 a$ & 94 & 90 & $R$ \\
\hline $18 \mathrm{~b}$ & 93 & 56 & $R$ \\
\hline
\end{tabular}

Scheme 5 
The possible mechanisms for the diethyl zinc addition to aldehydes by $\mathbf{1 7 b}$ or 18a are shown in Fig. 4. As in the models developed for chiral reduction of ketones, with $\mathbf{1 7} \mathbf{b}$, the diphenyl group exerts powerful steric effect on the transition state, thus favoring $\mathbf{D}$ over $\mathbf{D}^{\prime}$. Due to the steric factor of diphenyl group, the diethyl zinc reagents can only approach si face of the benzaldehyde. With 18a, the cyclohexyl group directs the course of the reaction favoring transition state $\mathbf{E}$ with $R e$ face attack of the zinc reagent.
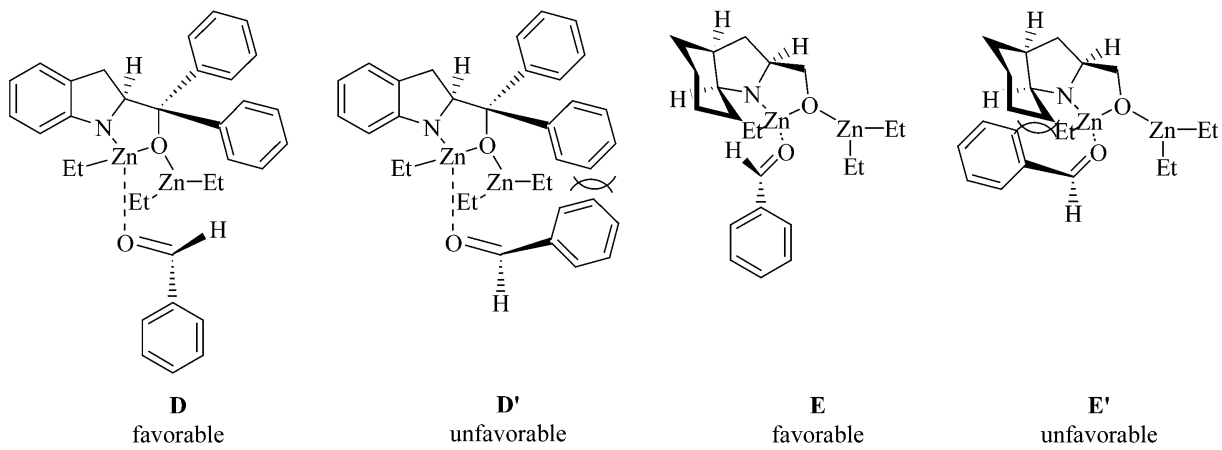

Fig. 4

In conclusion, starting from the same starting material, $(S)$-indoline-2-carboxylic acid, dual enantioselective control, the process that results in both $R$-and $S$-enantiomers in the same reaction system, has been achieved for the first time. Enantiopure $(S, S)$ - or $(R, R)$-2,3-dialkyltartaric acid and derivatives can be synthesized. Also, asymmetric Diels-Alder cyclizations of chiral amides with Lewis acids afforded extremely high diastereoselectivity of both opposite configuration of diastereomers depending on the Lewis used. Also, amino alcohols derived from $(S$ )-indoline-2-carboxylic acid were used as efficient catalysts in asymmetric reactions. They also exhibited dual enantioselective control in the asymmetric reduction of ketones and asymmetric alkylation of aldehydes. As shown in the examples above, careful design and selection of natural chiral sources, $(S)$-indoline-2-carboxylic acid, enabled the first dual enantioselective control in catalytic and stoichiometric asymmetric processes. Also, when compared with related $l$-proline derivatives, indoline derivatives not only showed dual enantioselective control, but also provided better reactivities. From these facts, it is apparent that efforts to develop new chiral ligands and auxiliaries should be directed toward dual enantioselective control and improved reactivity with the tool of rational design.

\section{REFERENCES}

1. (a) Y. H. Kim. Acc. Chem. Res. 34, 955 (2001); (b) Y. H. Kim, D. H. Park, I. S. Byun. J. Org. Chem. 58, 4511 (1993); (c) Y. H. Kim and J. Y. Choi. Tetrahedron Lett. 37, 5543 (1996).

2. S. M. Kim, I. S. Byun, Y. H. Kim. Angew. Chem., Int. Ed. Engl. 39, 728 (2000).

3. D. H. Park, S. H. Kim, J. D. Kim, Y. H. Kim. J. Chem. Soc., Chem. Commun. 963 (1999).

4. (a) T. Wirth. Angew. Chem., Int. Ed. Engl. 35, 61 (1996); (b) A. W. Konradi, S. J. Kemp, S. F. Pedersen. J. Am. Chem. Soc. 116, 1316 (1994); (c) B. Kammermeier, G. Beck, D. Jacobi, H. Jendralla. Angew. Chem., Int. Ed. Engl. 33, 685 (1994).

5. For reviews, see: (a) L. A. Paquett. Asymmetric Synthesis, Vol. 3, J. D. Morrison (Ed.), Academic Press, New York (1994); (b) W. Oppolzer. Angew. Chem., Int. Ed. Engl. 23, 876 (1984); (c) H. Waldmann. Synthesis 535 (1994).

6. Y. H. Kim, D. H. Park, I. S. Byun, I. K. Yoon, C. S. Park. J. Org. Chem. 56, 4511 (1993).

7. Y. H. Kim, D. H. Park, I. S. Byun. Heteroatom Chem. 3, 51 (1992). 
8. (a) S. Itsuno, M. Nakano, K. Ito, A. Hirao, M. Owa, N. Kanda, S. Nakahama. J. Chem. Soc., Perkin Trans. 12615 (1985); (b) E. J. Corey, R. K. Bakshi, S. Shibata, C. P. Chen, V. K. Singh. J. Am. Chem. Soc. 109, 7925 (1987); (c) I. K. Youn, S. W. Lee, C. S. Park. Tetrahedron Lett. 29, 4453 (1988); (d) J. Martens, Ch. Dauelsberg, W. Behnen, S. Wallbaum. Tetrahedron: Asymmetry 3, 347 (1992)

9. (a) E. J. Corey, R. K. Bakshi, S. Shibata. J. Am. Chem. Soc. 109, 5551 (1987); (b) V. Nevalinen. Tetrahedron: Asymmetry 2, 63 (1991).

10. (a) M. Kitamura, S. Suga, K. Kawai, R. Noyori. J. Am. Chem. Soc. 108, 6071 (1986); (b) K. Soai, A. Ookawa, T. Kaba, K. Ogawa. J. Am. Chem. Soc. 109, 7111 (1987); (c) E. J. Corey, P.-W. Yuen, F. J. Hannon, D. A. Wierda. J. Org. Chem. 55, 784 (1990). 